SYMPHYSEOTOMY FOR THE RELATIVE INDICATION, WITH COMPLICATIONS.

BY EDWARD P. DAVIS, A.M., M.D.,

Professor of Obstetrics in the Philadelphia Polyclinic; Clinical Professor of Obstetrics in the Jefferson Medical College.

AnTiseptic symphyseotomy for the accomplishment of difficult labor is too familiar to require defense, explanation or comment. ' $T$ wo unusual complications made the case in point interesting to me, and added observations of practical value to my experience with this operation.

The patient, a Polish woman, ignorant of English, was admitted to the Jefferson Maternity during the past spring in a pregnant condition. Through an interpreter, it was learned that her age was about forty. Her previous health had been good. Her sister had died after childbirth with a great swelling of the thigh and abdomen. On examination, the patient was fairly nourished. Her fetus, at the ninth month of pregvancy, was in the first position. She presented no apparent abnormality in the skeleton; her pelvic measurements were

Anterior Superior Iliac Spines Crests of Ilia Trochanters External Conjugate

Fetal heart sounds were strong and regular. 'The placenta was not discernible. Physical examination of the thorax and abdominal region revealed no abnor. mality. The patient's urine was that of a normal pregnant woman. By suprapubic manipulation the head was brought to the brim of the pelvis, presented in the right oblique pelvic diameter, and dipped slightly into the pelvis. During the time which elapsed between admission and confinement the patient's health was good, and she did housework without inconvenience.

Her labor was tedious, labor pains were good, the head presented in the right oblique pelvic diameter and partially engaged, Naegele's obliquity being more than normal. As exhaustion threatened, after dilatation was complete and all other means had been used to facilitate labor, the patient was etherized aud the membranes ruptured. While an assistant endeavored to promote the descent of the head by suprapubic pressure, Simpson's forceps was applied to the sides of the child's head, and axis-traction made with the Poullet tape attachment. A half-hour's intermittent traction failed to cause descent of the head, but increased the obliquity, the parietal bone of the right side presenting. No further efforts with forceps were made. As symphyseotomy was not expected, a pause of an hour ensued for suitable preparation and to secure instruments required. During this time the patient slept quietly.

She was again etherized, and symphyseotomy performed. The first complication of interest encountered was an abuormal height and ossitication of the pubic joint. 'The Galbiati knife, the chord of whose arc readily fits the height of the pubes in the average female skeleton, failed to grasp the joint. When a probe-pointed bistoury was used, the cartilage was found partly ossified. The joint was accordingly opened with a finger saw. The surfaces immediately separated two fingers' width, the child was readily delivered by forceps in the usual mechanism, and although asphyxiated, was resuscitated. The perineum was sutured, and a gauze drain carried behind the joint,

pressing the urethra back from the severed bones. This was removed after twenty-four hours, and the wound entirely closed.

'The patient's temperature ranged from $100^{\circ}$ to $102^{\circ}$ F. for the first week after delivery. Perineal and abdominal wounds healed promptly and well. Lactation was established, nutrition and excretion were well performed. The temperature then became normal, and 80 continued until the $21 \mathrm{st}$ day. It is my custom to allow symphyseotomy patients to first turn upon the side three weeks after the operation, and then with the assistance of a nurse. As the patient could not understand perfectly what was said, she eluded the nurses, and while a labor in an adjoining ward was in progress, turned suddenly over. This was followed by thrombosis of the femoral veins upon the left side, accompanied by swelling, pain especially in the popliteal space, and temperature of $102^{\circ} \mathrm{F}$. So far as the genital organs were concerned there was no evidence of infection or inflammation revealed by examination. The patient's nervous system was unduly disturbed by this condition, as she stated to an interpreter that her sister had died under a similar condition. Ten days after the occurrence of the thrombosis, her temperature was normal ; she was able to gradually get up and to walk carefully. Three months after delivery a thorough examination of the patient revealed no diseased condition; she was doing light housework. Examination of her pelvis revealed an increase in the internal conjugate of one and one-half centimetres following the symphyseotomy. Good union existed at the pubes. The uterus and pelvic floor were in normal condition. The measurements of the child's head were as follows :

Maximum diameter

Occipito-frontal

Fronto-mental

Suboccipito-bregmatic

Bi-parietal

Bi-temporal

Bis-acromial

Length

Weight

Sex, female.

The child's head, which was distorted by efforts to deliver, acquired its normal contour. It nursed well for a week, when it developed obscure symptoms of inanition, persistence of meconium stools, and failure of strength. Death occurred on the eighth day. Postmortem examination revealed congenital absence of the common bile duct; no other cause for death could be found.

In undertaking symphyseotomy in women older than thirty, the operator must not trust wholly to a knife; this is the second case in my experience in which a condition of partial ossification has resisted the Galbiati knife and probe-pointed bistoury. The occurrence of thrombosis during convalescence may be explained by the dislodgement of septic thrombi in the uterine sinuses, although it is not perfectly clear how such infected thrombi could cause no disturbance before the patient turned. A more rational explanation to me lies in mechanical injury in the sudden turn done to vessels predisposed to thrombosis by long recumbent posture, a retention dressing about the hips, and recovery from a surgical procedure of moderate severity. 'There was no evidence of abscess in any part of the thighs, which might readily have followed septic thrombosis. This case illustrates well the im- 
portance of good nursing after symphyseotomy, without which this patient's complicated labor would have been far more dangerous and trying. ${ }^{1}$

The patient's pelvic measurements were such that delivery, possibly with forceps, was all that was anticipated; symphyseotomy was performed for the relative indication. The case impressed upon the operator and upon the class in clinic the futility of attempting to deliver a living child by forceps without symphyseotomy, when a relative disproportion in size between head and pelvis sufficient to prevent engagement exists.

\section{A STUDY OF ANEMIA CASES WITH THE AID OF THE HEMOMETER AND BLOOD- COUNTER.'}

BERVICE OF A. K. STONE, M.D.

REPOR'TED BY ANNA G. RICHARDSON, M.D., ELLIOT P. JOSLIN, PH.B. AND FRANCIS P. DENNY, A.B.

Last summer, through the kindness of Dr. Stone, Mr. Joslin and Mr. Denny, of the Harvard Medical School, and I, were allowed to study with him all the cases of anemia that came to the Woman's Clinic OutPatient Department of the Massachusetts General Hospital.

The method of examination was as follows: Every patient who showed any signs of anemia, first had her blood examined for hemoglobin. We used the instrument of von Fleischl. Then if the percentage of hemoglobin was low, the corpuscles were counted by $\mathrm{Mr}$. Joslin or Mr. Denny. The instrument used was the Thoma-Zeiss. As long as the patients could be kept under observatiou, this was repeated from time to time. The counting and hemoglobin test was always made by the same person for each case, so as to eliminate error as much as possible, the personal error being presumably always the same.

In some of the cases in which the diagnosis was obscure, slides were prepared and stained, and the white corpuscles studied by Mr. Dolliver of the Medical School.

The hemoglobin of 168 patients was examined, all of whom presented symptoms of anemia. Of these 8 were above 100 per cent., the highest being 105 per cent.; 19 were between 90 and 100 per cent.; and considering 75 per cent. as the dividing line between the anemic and the not anemic, 89 were not anemic, that is to say, by the ordinary method, the diagnosis was incorrect in 53 per cent. of the cases. These mistakes in diagnosis were evenly distributed throughout the service. The hemoglobin of a number of persons in health was examined, and it ranged from 70 per cent. to more than the hemometer would register. We found that in the anemic, and in the non-anemic, the amount of color in the skin and mucous membranes was of no value in estimating the amount of hemoglobin.

Owing to the difficulty in an out-patient department of keeping track of the patients, and to the fact that many come to the Massachusetts General Hospital simply for diagnosis, there were about 33 of these anemic cases seen a sufficient number of times, and over a long enough period, to make the cases of value. Of these, 20 were Americans; 5 were from the British

1 Read before the Medical Section of the Suffolk District Medical Society, March 21, 1894 .

1 Those interested in this subject are referred to an article by my chief nurse, Miss Russell (The Trained Nurse, July, 1894, "Nursing chief nurse, Miss Russell (Th
of Symphyseotomy Cases ").
Provinces; 5 were Irish; and 1 each Russian, German and Swiss. The large number of Americans may be due to the fact that the patients who go to the Massachusetts General Hospital are generally of a better class, although many of the Americans were of foreign parentage. With regard to age, 22 were between fifteen and twenty years, 5 between twenty and twenty-five years; and of the six over twenty-five years, 4 were secondary anemias, and one a leukemia. Although 22 were under twenty years of age, there were ouly 3 school-girls, and a number of them had been at work for more than three or four years. Twelve did housework; 5 worked in shops; 3 were seamstresses, and a number were unable to work at all. The number of cases doing housework is large. This is probably due to the lack of fresh air, and the attendant evils of a poor appettite and a bad diet.

The symptoms were such as are usually given: pains; usually in the head, often in some part of the chest; palpitation; dyspnea; and anorexia. Hemic heart-murmurs and a venous hum in the cervical vessels were present in a large number of cases. There was one case of amenorrhea. Menstruation was scanty in about half of the cases and normal in the rest. Constipation was a symptom in 16 of the cases, but very probably this was true with more of them. There was diarrhea in two cases; in one of these the diarrhea was probably the cause of the anemia. The diarrhea was stopped; and the corpuscles increased from $2,160,000$ to $4,584,000$ in a few days, and the hemoglobin dropped from 35 to 28 per cent. Then this was steadily increased to the normal by giving the patient Blaud's pills.

One case, which we suspected was of syphilitic origin, did not improve until put on potassium iodide with the iron.

Another case, age 34, in which the hemoglobin was 35 per cent., the number of corpuscles between three and one-half and four million, and the symptoms very severe, did not improve at all in three months of faithful following of directions as to fresh air, food, etc., and iron and Fowler's solution for medicine. Finally, in December (she was first seen in July) the hemoglobin had increased to 43 per cent., and the number of corpuscles to five and one-half million. This patient was seeu on March 5th, when the hemoglobin was found to be 54 per cent. It was thought at first that it might be a case of progressive peruicious anemia; but the examination of the stained specimen did not bear out the diagnosis, and the later improvement also excluded this diagnosis. There was at times a slight trace of albumin in the urine. She had also a cough, but no expectoration, and the examination of the lungs was negative.

In another case, a woman twenty.eight years old gave the history of having had her first child before she was sixteen years old, and ten pregnancies since, in six of which she aborted. The youngest, a child of four months, she was nursing. This woman had the lowest record of all for hemoglobin, it being but fifteen per cent., and the number of red blood corpuscles was two aud one-half million. She weaned her baby, and was put on liq. potass. arsenitis, which was later changed to tincture of the chloride of iron. In four months of constant treatment she only increased to 23 per cent. of hemoglobin and four and one-half million of corpuscles. When seen two months later, she had just menstruated for the first time since the birth of her last 